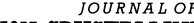

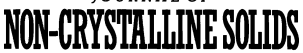

\section{Time-resolved study of photoluminescence polarization in a-C:H films}

\author{
M.N. Berberan-Santos ${ }^{\mathrm{a}, *}$, A. Fedorov ${ }^{\mathrm{a}}$, T. Heitz $^{\mathrm{b}}$, C. Godet $^{\mathrm{b}}$, J.E. Bourée ${ }^{\mathrm{b}}$, \\ J.P. Conde ${ }^{\mathrm{c}}$ \\ a Instituto Superior Técnico, Centro de Química-Física Molecular, Av. Rovisco Pais, 1049-001 Lisbon, Portugal \\ ${ }^{\mathrm{b}}$ Laboratoire de Physique des Interfaces et des Couches Minces (UMR 7647 CNRS), Ecole Polytechnique, 91128 Palaiseau, France \\ ${ }^{\mathrm{c}}$ Instituto Superior Técnico, Department of Materials Engineering, Av. Rovisco Pais, 1049-001 Lisbon, Portugal
}

\begin{abstract}
The decays of intensity and polarization anisotropy of the photoluminescence (PL) of polymer-like amorphous carbon films have been measured at 300 and $80 \mathrm{~K}$ using 4.13 and $2.15 \mathrm{eV}$ excitation. For emission energies, $E_{\text {em }}$, between 1.8 and $3.5 \mathrm{eV}$, PL polarization anisotropy decreases within 100 ps and reaches a plateau within $1 \mathrm{~ns}$. The anisotropy plateau increases from 0.02 to 0.12 and the intensity decay time decreases from $1 \mathrm{~ns}$ to $40 \mathrm{ps}$ as $E_{\mathrm{em}}$ increases. Anisotropy and intensity decays are interpreted within a dipole-dipole energy transfer (Förster) model. (c) 2000 Elsevier Science B.V. All rights reserved.
\end{abstract}

\section{Introduction}

In contrast to amorphous silicon films, intense steady-state photoluminescence (PL) from wide bandgap polymer-like amorphous carbon $(\mathrm{a}-\mathrm{C}: \mathrm{H})$ has been observed in the visible range, independent of temperature [1-3] and with no correlation between the paramagnetic defect state density and the PL efficiency [4]. These differences have been attributed to a faster radiative recombination in a$\mathrm{C}: \mathrm{H}$, where photo-induced electron-hole pairs may recombine as a neutral entity made of two carriers bound by a Coulomb interaction. Indeed, exciton-like resonance features have been detected in PL excitation spectra of a-C:H films [3].

In a-C:H, the polarization memory (anisotropy) of steady-state PL excited using linearly polarized

\footnotetext{
${ }^{*}$ Corresponding author.
}

light $[5,6]$ has been taken as evidence of recombination of localized electron-hole pairs. Furthermore, time-resolved measurements have shown that the PL polarization anisotropy of C-rich a- $\mathrm{Si}_{x} \mathrm{C}_{1-x}$ : $\mathrm{H}$ alloys is constant over nanoseconds at $4 \mathrm{~K}$ and decreases on a nanosecond timescale at $300 \mathrm{~K}$ [7].

This paper presents time-resolved PL intensity and polarization anisotropy decays in the ps to ns range as a function of emission energy, $E_{\mathrm{em}}$. The dependence of PL properties on temperature $(80-300 \mathrm{~K})$ and excitation energy, $E_{\text {exc }}$, is also measured. Polarization anisotropy and intensity decays are interpreted within a dipole-dipole energy transfer model.

\section{Experimental}

Carbon films were deposited at $70^{\circ} \mathrm{C}$ on the RF electrode of a radiofrequency-assisted microwave 
reactor [8] using $n$-butane as a carbon precursor injected in the microwave (MW) post-discharge. The typical polymer-like carbon film chosen for this study was grown on single crystal silicon (c-Si) to avoid PL from the substrate. The energy at which the absorption coefficient is $\alpha=10^{4} \mathrm{~cm}^{-1}$ is $3.45 \pm 0.05 \mathrm{eV}$.

Time-averaged PL measurements were performed at $300 \mathrm{~K}$ with a SPEX Fluorolog system. Using ultraviolet-excitation (at $300 \mathrm{~nm}$ with $27 \mathrm{~nm}$ excitation bandwidth), polarized spectra were recorded in the $\mathrm{V}-\mathrm{V}, \mathrm{V}-\mathrm{H}, \mathrm{H}-\mathrm{V}$ and $\mathrm{H}-\mathrm{H}$ con- figurations with a $20 \mathrm{~s}$ integration time. The photomultiplier background level was subtracted before calculation of the time-averaged anisotropy, $R_{\mathrm{SS}}\left(-0.2 \leqslant R_{\mathrm{SS}} \leqslant 0.4\right)$

$$
\begin{aligned}
R_{\mathrm{SS}} & =\left(I_{/ /}-I_{\perp}\right) /\left(I_{/ /}+2 I_{\perp}\right) \\
& =\left(I_{\mathrm{VV}} / k-I_{\mathrm{VH}}\right) /\left(I_{\mathrm{VV}} / k+2 I_{\mathrm{VH}}\right),
\end{aligned}
$$

where $k=\left(I_{\mathrm{HV}} / I_{\mathrm{HH}}\right)$ is introduced to take into account the polarization response of the monochromator.
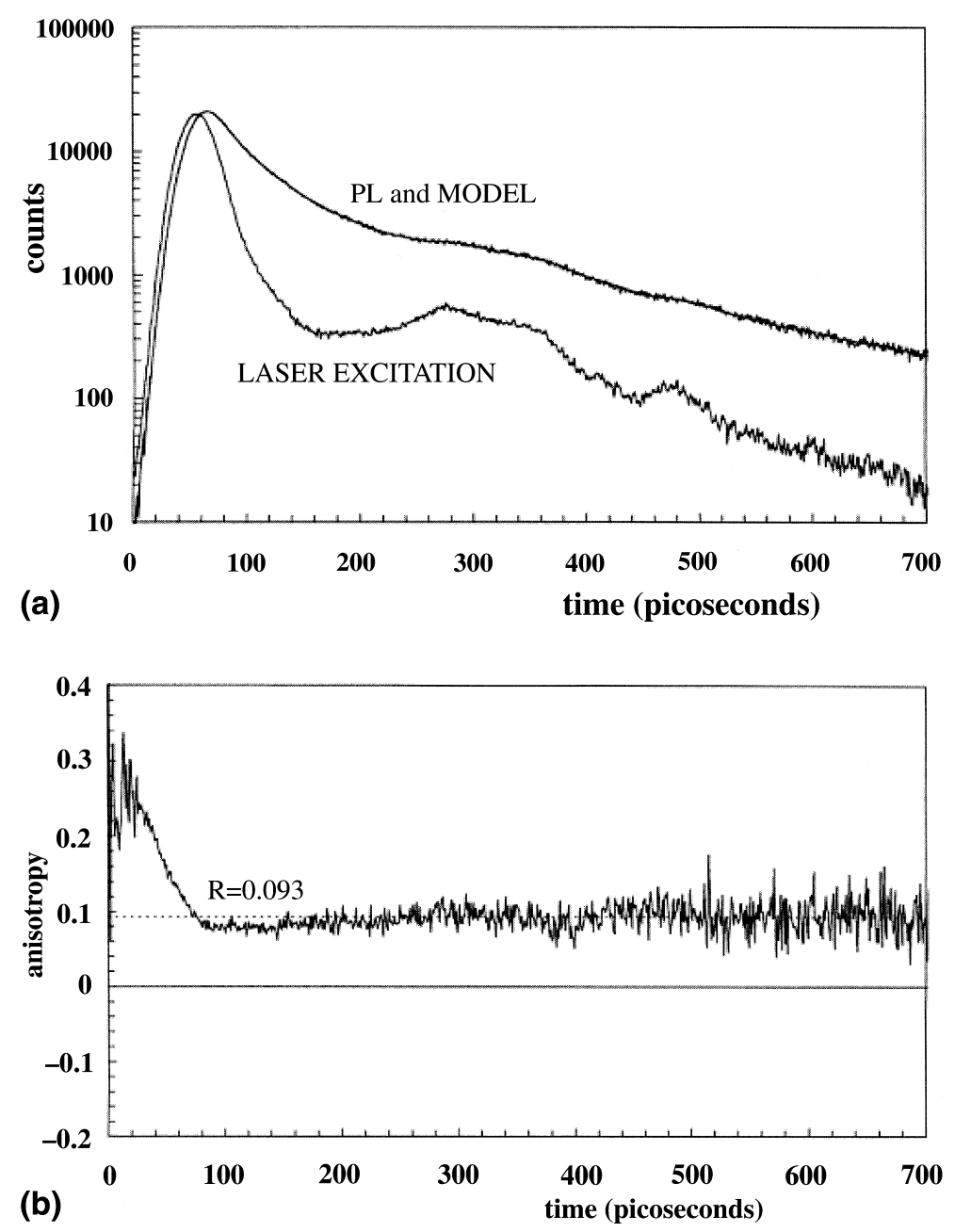

Fig. 1. (a) PL intensity decay at $E_{\mathrm{em}}=2.95 \mathrm{eV}$ using $E_{\mathrm{exc}}=4.13 \mathrm{eV}$ excitation (the time-dependence of the laser excitation is also shown). The fitted 3-exponential decay is superimposed to experimental data. (b) Anisotropy decay: the plateau value $(R=0.093)$ is fitted between 200 and 700 ps. 
Table 1

PL characteristics of amorphous carbon: time-averaged anisotropy $R_{\mathrm{SS}}$ (measured with a $\lambda_{\mathrm{em}}$ width of $9 \mathrm{~nm}$ ), intensity decay time $\tau_{\mathrm{I}}$ and anisotropy plateau value $R(1 \mathrm{~ns}) . E_{\text {exc }}=4.13 \mathrm{eV}(300 \mathrm{~nm})$

\begin{tabular}{lllll}
\hline$\lambda_{\mathrm{em}}(\mathrm{nm})$ & $E_{\mathrm{em}}(\mathrm{eV})$ & $\tau_{\mathrm{I}}(\mathrm{ps})$ & $R(1 \mathrm{~ns})^{\mathrm{a}}$ & $R_{\mathrm{SS}}$ \\
\hline 350 & 3.54 & 39 & 0.120 & $0.25^{\mathrm{b}}$ \\
420 & 2.95 & 90 & 0.093 & 0.258 \\
470 & 2.64 & 204 & 0.080 & 0.127 \\
545 & 2.28 & 541 & 0.041 & 0.076 \\
700 & 1.77 & 965 & 0.025 & $0.035^{\mathrm{b}}$ \\
\hline
\end{tabular}

${ }^{\mathrm{a}} R(1 \mathrm{~ns}) \pm 0.01$.

${ }^{\mathrm{b}}$ Extrapolated values \pm 0.05 .

The time-resolved PL was measured at $300 \mathrm{~K}$ using laser excitation at $4.13 \mathrm{eV}(300 \mathrm{~nm})$ and 2.15 eV $(577 \mathrm{~nm})$. In the latter case, complementary data were collected at $80 \mathrm{~K}$. Picosecond luminescence decays were obtained by the single-photon timing method with excitation from a dye laser (Coherent 701-2 ) delivering $\sim 4$ ps pulses $(\sim 40 \mathrm{~nJ} /$ pulse) at a frequency of $3.4 \mathrm{MHz}$. The instrument response function has an effective full width at half maximum of $30 \mathrm{ps}$. Intensity decay measurements were made by alternating collection of impulses and decays with the emission polarizer set either at magic angle position (unpolarized PL) or at $\mathrm{H}$ (respectively V) position. Detection by a microchannel plate photomultiplier was done by passing the emission through a depolarizer and then through a monochromator [9].

\section{Results}

Fig. 1 shows typical experimental decays of intensity (Fig. 1(a)) and polarization anisotropy (Fig. 1(b)) for PL emission at $E_{\text {em }}=2.95 \mathrm{eV}(420$ $\mathrm{nm})$ excited at $E_{\mathrm{exc}}=4.13 \mathrm{eV}(300 \mathrm{~nm})$. Intensity decays have been fitted using a convolution between the laser excitation and an intrinsic decay function made of the sum of three exponentials (six parameter fit) [9]. This empirical analytic function is found to describe accurately the PL decays in a-C:H films and silicon-carbon alloys [10]. The intrinsic decay times $\tau_{\mathrm{I}}$ ) of PL intensity, obtained from a weighted average of the fitted decay times, decrease from 965 to $39 \mathrm{ps}$ as a function of increasing $E_{\mathrm{em}}$ (Table 1).
As illustrated in Fig. 1(b), for $4.13 \mathrm{eV}$ excitation, PL polarization anisotropy decreases from $\sim 0.25$ within about $100 \mathrm{ps}$, independently of $E_{\mathrm{em}}$. Within $300-1000 \mathrm{ps}$, the anisotropy reaches a plateau with a finite value - noted $R(1 \mathrm{~ns})$ - which increases from 0.02 to 0.12 as a function of increasing $E_{\text {em }}$. Plateaus as large as $0.21 \pm 0.02(300 \mathrm{~K})$ or $0.24 \pm 0.03(80 \mathrm{~K})$ have been obtained for $E_{\mathrm{em}}=$ $1.75 \mathrm{eV}$, excited at lower energy $E_{\mathrm{exc}}=2.15 \mathrm{eV}$.

For emission energies, $E_{\mathrm{em}},<2.7 \mathrm{eV}$, the total decay time $\left(\tau_{\mathrm{I}}\right.$ in Table 1$)$ is large as compared to the depolarization time $\left(\tau_{\mathrm{DP}} \sim 50 \mathrm{ps}\right)$ and the radiative recombination is weakly polarized, whereas $\tau_{\mathrm{I}} \sim \tau_{\mathrm{DP}}$ for higher $E_{\mathrm{em}}$ values $\left(E_{\mathrm{em}} \geqslant 3.0 \mathrm{eV}\right)$ corresponding to strongly polarized emission.

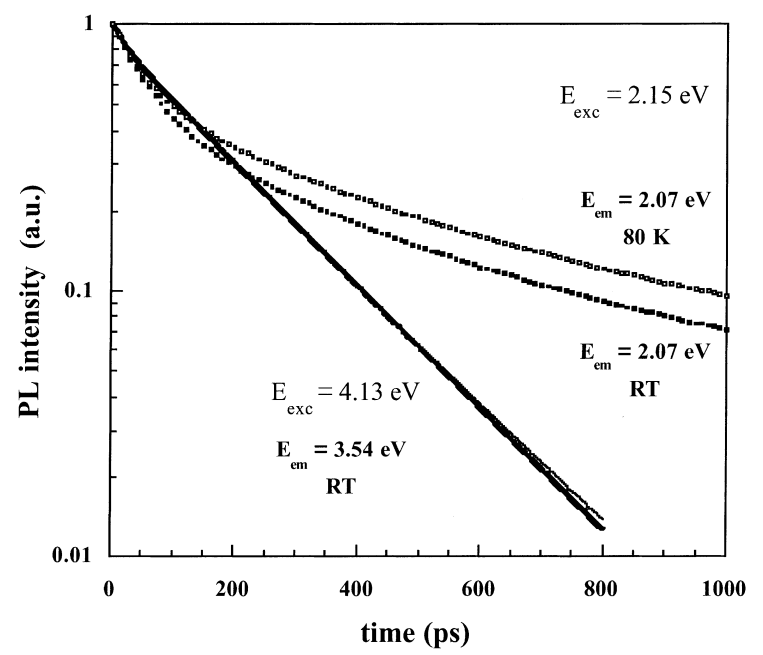

Fig. 2. PL intensity decay at $E_{\mathrm{em}}=2.95 \mathrm{eV}$ using $E_{\mathrm{exc}}=4.13$ $\mathrm{eV}$ (at $300 \mathrm{~K}$ ) with the fitted Förster decay superimposed to experimental data. PL intensity decay at $E_{\mathrm{em}}=2.07 \mathrm{eV}$ using $E_{\text {exc }}=2.15 \mathrm{eV}$ (at 80 and $300 \mathrm{~K}$ ). 
In agreement with Rusli et al. [5], a monotonic increase of $R_{\mathrm{SS}}$ as a function of increasing emission energy, $E_{\text {em }}$, in the range $2.2-3.0 \mathrm{eV}$ is reported in Table 1. The subnanosecond decay times $\left(\tau_{I}\right)$, due to both radiative and non-radiative recombination mechanisms, are in quantitative agreement with previous studies of amorphous carbon films [11]. A similar dependence of intrinsic decay times on $E_{\text {em }}$ has been reported in C-rich silicon carbon alloys [10].

To assess the role of excitation energy and measurement temperature on carrier relaxation, intrinsic decay rates were measured at 300 and $80 \mathrm{~K}$. For $E_{\mathrm{em}}$ values between 1.75 and $2.07 \mathrm{eV}$, the decay rates are found to be independent of measurement temperature $(0.78-0.92$ ns at $80 \mathrm{~K}$, $0.66-0.78 \mathrm{~ns}$ at $300 \mathrm{~K}$, using $E_{\text {exc }}=2.15 \mathrm{eV}$ ) and on excitation energy $(0.7-1 \mathrm{~ns}$ at $2.15 \mathrm{eV}, 0.96 \mathrm{~ns}$ at $4.13 \mathrm{eV}$, measured at $300 \mathrm{~K}$ ). Fig. 2 illustrates the lack of temperature dependence of intensity decays in the ps to ns range, for $E_{\mathrm{em}}=2.07 \mathrm{eV}$ using $E_{\text {exc }}=2.15 \mathrm{eV}$.

\section{Discussion}

A possible depolarization mechanism is incoherent radiationless excitation transfer from a donor to an acceptor chromophore via dipole-dipole interactions (Förster mechanism) [12]. For each transfer, the depolarization efficiency is about $96 \%$. The depolarization rate, $v$ is proportional to the energy overlap between the emission spectral density of donor sites and the absorption spectrum of acceptor sites, separated by a distance $R$, and can be expressed as

$v=\left(1 / \tau_{\mathrm{I}}\right)\left(R_{0} / R\right)^{6}$,

where the critical Förster radius, $R_{0}$, is usually between 2 and $6 \mathrm{~nm}$. Assuming a random distribution of chromophores, the decay law of initially excited donors is known [12]: $i(t)=\exp \left[-\left(t / \tau_{\mathrm{F}}\right)-b t^{1 / 2}\right]$, where $b=0.845 n_{\mathrm{A}}\left[\pi / \tau_{\mathrm{F}}\right]^{1 / 2}$ and $n_{\mathrm{A}}=4 / 3 \pi N_{\mathrm{A}}\left[R_{0}\right]^{3}$ is a number of acceptor molecules within a sphere of radius $R_{0}$. A fit of this equation to the decay at $3.54 \mathrm{eV}$ (Fig. 2) gives $\tau_{\mathrm{F}}=214 \mathrm{ps}$ (decay time of the primarily excited blue chromophores) and $b=0.019 \mathrm{ps}^{-1 / 2}$, which correspond to densities, $N_{\mathrm{A}}$, of acceptor molecules between $2 \times 10^{17}$ and $6 \times 10^{18} \mathrm{~cm}^{-3}$.

In this model, the long time plateau of the anisotropy at energy $E_{\mathrm{em}}$ is due to the fraction of chromophores which are isolated. Using $R(1 \mathrm{~ns})$ $=0.12$ at $E_{\mathrm{em}}=3.54 \mathrm{eV}$, we obtain an estimate for the fraction of isolated chromophores, $p=$ $0.12 / 0.40=0.3$, since 0.4 is the maximum polarization anisotropy. Assuming a random distribution, the probability that no nearest neighbors occur for distances smaller than $R$ is given by $p=\exp \left(-4 / 3 \pi N_{\mathrm{A}} R^{3}\right)$, resulting in $N_{\mathrm{A}}=$ $-3 \ln p / 4 \pi R^{3}$. Non-radiative transfer will be negligible for $R \geqslant 2 R_{0}$, corresponding to distances of 4-12 nm, depending on $R_{0}$. From the above numbers, we obtain concentrations of acceptors between $1.6 \times 10^{17}$ and $4.5 \times 10^{18} \mathrm{~cm}^{-3}$ in agreement with the previous estimate.

\section{Conclusions}

Time-resolved PL decays in a-C:H have been interpreted using a radiationless energy transfer mechanism. In this model, we expect that: (i) The PL anisotropy will be present at all emission energies from the directly excited larger energy chromophores (donors), the only ones to have polarized emission. (ii) The anisotropy plateau at longer times is due to the contribution to the PL of directly excited chromophores that have no acceptors in their neighborhood. We expect this contribution to be smaller at lower emission energies where the emission of acceptors is dominant. (iii) Increasing excitation energy selects chromophores higher in the bandtails, providing a greater density of neighboring chromophores that can act as acceptors. In this case, the anisotropy decay is faster and the plateau value is smaller. In contrast, excitation at lower energies selects donors that are surrounded by chromophores that cannot, in part, act as acceptors because their energies are likely to be higher than the primarily excited donors. In this way, the plateau should be larger. All the described trends are observed. As predicted by the model, the decay rate of anisotropy either coin- 
cides or is faster than the decay rate of high energy donors.

\section{Acknowledgements}

We are grateful to V. Chu (INESC, Lisbon) who contributed to the steady-state PL measurements. This work was supported by a grant from the CNRS-ICCTI exchange program and by the project PRAXIS 3/3.1/MMA/1901/95.

\section{References}

[1] S.V. Chernyshov, E.I. Terukov, V. Vassilyev, A.S. Volkov, J. Non-Cryst. Solids 134 (1991) 218.

[2] J. Robertson, Phys. Rev. B 53 (1996) 16302.
[3] T. Heitz, C. Godet, J.E. Bourée, B. Drévillon, J.P. Conde, Phys. Rev. B 60 (1999) 6045.

[4] S. Schütte, S. Will, H. Mell, W. Fuhs, Diamond Related Mater. 2 (1993) 1360.

[5] Rusli, G.A.J. Amaratunga, J. Robertson, Phys. Rev. B 53 (1996) 16306.

[6] M. Koós, I. Pócsik, L. Tóth, J. Non-Cryst. Solids 164-166 (1993) 1151.

[7] Y. Masumoto, H. Kunitomo, S. Shionoya, H. Munekata, H. Kukimoto, Solid State Commun. 51 (1984) 209.

[8] C. Godet, T. Heitz, J.E. Bourée, B. Drévillon, C. Clerc, J. Appl. Phys. 84 (1998) 3919.

[9] A. Fedorov, M.N. Berberan-Santos, J.P. Lefèvre, B. Valeur, Chem. Phys. Lett. 267 (1997) 467.

[10] J.P. Conde, V. Chu, M. da Silva, A. Kling, Z. Dai, J.C. Soares, S. Arekat, A. Fedorov, M.N. Berberan-Santos, F. Giorgis, C.F. Pirri, J. Appl. Phys. 85 (1999) 3327.

[11] W. Lormes, M. Hundhausen, L. Ley, J. Non-Cryst. Solids 227 (1998) 570.

[12] M.D. Galanin, Luminescence of Molecules and Crystals, Cambridge International Science, Cambridge, 1996. 